\title{
Cadmium isotope variations in chondrites and acid leachates of Jbilet Winselwan (CM2)
}

\author{
E. R. Toth ${ }^{1 *}$, M. A. FeHR ${ }^{1}$, M. FrIEBEL ${ }^{1}$ AND M. \\ SCHÖNBÄCHLER ${ }^{1}$
}

${ }^{1}$ Institute of Geochemistry and Petrology, ETH Zürich,

Switzerland (*correspondence: eniko.toth@erdw.ethz.ch)

Refractory elements show well-resolved nucleosynthetic isotope variations at the bulk meteorite scale (e.g. Ti, $\mathrm{Zr}, \mathrm{Pd}$ [1-3]), contrary to the homogeneous compositions displayed by moderately volatiles (e.g. $\mathrm{Zn}, \mathrm{Se}, \mathrm{Te}$ [4-6]). Cadmium is a prime candidate to further evaluate this trend due to its moderately volatile nature and eight stable isotopes generated by a variety of nucleosynthetic processes. Furthermore, it is a potential tool to constrain the exposure histories of stony meteorites because ${ }^{113} \mathrm{Cd}$ has a large neutron capture crosssection. This study reports $\mathrm{Cd}$ isotope data for seven bulk chondrites and acid leachates of Jbilet Winselwan (CM2) to search for nucleosynthetic isotope variations and thermal neutron capture effects.

Cadmium isotopes were analysed by MC-ICPMS $(\mathrm{Nu}$ Plasma II) and internally normalised to ${ }^{116} \mathrm{Cd} /{ }^{111} \mathrm{Cd}[7,8]$. In addition to high-precision analyses, data were also obtained for samples with low Cd contents, such as leachates $(\leq 2 \mathrm{ng}$ Cd) and GRA 06100 (0.7 ng Cd) [8].

Bulk carbonaceous and enstatite chondrites measured at high-precision and all acid leachates of Jbilet Winselwan display nucleosynthetic $\mathrm{Cd}$ isotope homogeneity [8]. The $\mathrm{Cd}$ depleted CR2 chondrite GRA 06100 shows hints of nucleosynthetic $\mathrm{Cd}$ isotope variations that are, however, only marginally resolved [8]. Analyses of such low Cd contents are analytically challenging [8]. A recent model proposed that the magnitude of nucleosynthetic isotope variations of elements in the mass region of Pd depend largely on their condensation behaviour around AGB stars and thus their volatility [3]. The lack of well-resolved nucleosynthetic $\mathrm{Cd}$ isotope variations is in good agreement with this model.

Small variations of up to $-0.6 \pm 0.3$ in $\varepsilon^{113} \mathrm{Cd}$ related to thermal neutron capture effects were observed for Jbilet Winselwan, Indarch, Allende and MIL 090001 [8]. This renders $\mathrm{Cd}$ a new potential thermal neutron capture monitor for chondrites with exposure ages exceeding a few Ma [8].

[1] Trinquier et al. (2009) Science 324, 374-376. [2] Akram et al. (2015) GCA 165, 484-500. [3] Ek et al. (2020) Nat. Astron. [4] Moynier, Dauphas \& Podosek (2009) ApJ 700, L92-L95. [5] Labidi et al. (2018) EPSL 481, 212-222. [6] Fehr et al. (2005) GCA 69, 5099-5112. [7] Friebel et al. (2020) JAAS 35, 273-292. [8] Toth et al. (2020) GCA. 